\title{
Nanoscale pillar hypersonic surface phononic crystals
}

\author{
D. Yudistira, ${ }^{1,2, *}$ A. Boes, ${ }^{1,2}$ B. Graczykowski, ${ }^{3}$ F. Alzina, ${ }^{3}$ L. Y. Yeo, ${ }^{4}$ C. M. Sotomayor Torres,,${ }^{3,5}$ and A. Mitchell ${ }^{1,2}$ \\ ${ }^{1}$ School of Engineering, RMIT University, VIC 3001, Melbourne, Australia \\ ${ }^{2}$ ARC Centre of Excellence for Ultra-High Bandwidth Devices for Optical Systems (CUDOS), RMIT University, \\ VIC 3001, Melbourne, Australia \\ ${ }^{3}$ Catalan Institute of Nanoscience and Nanotechnology (ICN2), CSIC and The Barcelona Institute of Science and Technology, Campus UAB, \\ Bellaterra, E-08193 Barcelona, Spain \\ ${ }^{4}$ Micro/Nanophysics Research Laboratory, RMIT University, VIC 3001, Melbourne, Australia \\ ${ }^{5}$ ICREA, Pg. Lluís Companys 23, E-08010 Barcelona, Spain
}

(Received 28 June 2016; revised manuscript received 28 August 2016; published 15 September 2016)

\begin{abstract}
We report on nanoscale pillar-based hypersonic phononic crystals in single crystal Z-cut lithium niobate. The phononic crystal is formed by a two-dimensional periodic array of nearly cylindrical nanopillars $240 \mathrm{~nm}$ in diameter and $225 \mathrm{~nm}$ in height, arranged in a triangular lattice with a 300-nm lattice constant. The nanopillars are fabricated by the recently introduced nanodomain engineering via laser irradiation of patterned chrome followed by wet etching. Numerical simulations and direct measurements using Brillouin light scattering confirm the simultaneous existence of nonradiative complete surface phononic band gaps. The band gaps are found below the sound line at hypersonic frequencies in the range $2-7 \mathrm{GHz}$, formed from local resonances and Bragg scattering. These hypersonic structures are realized directly in the piezoelectric material lithium niobate enabling phonon manipulation at significantly higher frequencies than previously possible with this platform, opening new opportunities for many applications in plasmonic, optomechanic, microfluidic, and thermal engineering.
\end{abstract}

DOI: 10.1103/PhysRevB.94.094304

\section{INTRODUCTION}

Phononic crystals are artificially engineered structures made of composite materials of significantly different acoustic impedances in a periodically ordered assembly. Typically, these structures consist of pillar and/or hole inclusions confined within a planar matrix. The configuration of these arrays gives rise to a phonon band structure due to local resonances [1-7] and Bragg scattering [8-13]. The band structures commonly exhibit phononic band gaps [3,4,7,10-13] and can be engineered to achieve negative refraction [14-16] with the potential for confinement and manipulation of acoustic waves at scales commensurate with their wavelengths or even subwavelengths [16-19]. To date, research has primarily been focused on the development of phononic crystals which operate in the subgigahertz frequency regime through the incorporation of periodic inclusions at millimeter (sound) to micrometer (ultrasound) scales [3,4,10-13,20] for various applications that include acoustic filters, acoustic lenses, sensors, and microfluidics.

Very recently, however, there has been a growing interest in hypersonic phononic crystals, exhibiting features at the submicron scale that allow the manipulation of acoustic phonons at $\mathrm{GHz}$ to $\mathrm{THz}$ frequencies. Such structures will find utility in future broadband wireless communications and also optomechanic and plasmonic coupling in micro- and nanostructures [21-27] in the $\mathrm{GHz}$ regime and potentially even manipulation of thermal phonons (heat) for engineering thermal conductivity and other thermoelectric properties of materials for energy harvesting applications as the $\mathrm{THz}$ regime becomes accessible [28-31]. If it is possible to overcome the current $1-\mathrm{GHz}$ frequency ceiling in acoustically driven

*didit.yudistira@rmit.edu.au microfluidics [32,33], then it should be possible to achieve nanofluidic actuation facilitating biomolecular manipulation and sensing at single cell and even single molecule levels.

With recent advances in nanofabrication, especially in CMOS silicon platforms, reports have emerged of hypersonic phononic crystals for bulk [8,9], plate [7,21,23], and surface wave phononics [34]. Nanophotonics and thermoelectric applications exploiting hypersonic phonons in silicon have also been recently demonstrated [21-23,28-30]. While silicon offers a mature fabrication route for realizing nanoscale phononic crystals, many of the inherent properties of silicon make full exploitation of the material for phononic applications and its coupling with photonics challenging.

Lithium niobate $\left(\mathrm{LiNbO}_{3}\right)$ has long been the industry standard workhorse of both phononics and photonics given its attractive piezoelectric, electro-optic, and acousto-optic properties and ability to support optical and surface acoustic waves with very low losses across a broad frequency range [35,36]. A monolithic phononic crystal in $\mathrm{LiNbO}_{3}$ such as that demonstrated previously [37,38], even though at lower frequencies, is highly attractive, for example, in microfluidic devices, since it circumvents the need to couple the acoustic energy through fluid couplants into phononic crystal structures in superstrates [39] which can incur considerable energy loss.

Unlike silicon, however, creating structures at the microscale and below in $\mathrm{LiNbO}_{3}$ has always been a significant challenge especially for the high aspect ratios required to prevent radiation of high frequency surface phonons in surface acoustic wave phononic crystals [40]. Much research effort has therefore been invested into developing techniques for etching $\mathrm{LiNbO}_{3}$. These include wet etching with hydrofluoric acid (HF) [41-43] as well as dry etching using masked ion milling and direct write focused ion beam (FIB) milling [44], reactive ion etching $[45,46]$, and plasma enhanced etching [40]. The latter dry etching techniques, in particular, have been shown 
to be effective for producing submicron structures, although redeposition of $\mathrm{LiF}$ and $\mathrm{NbF}_{x}$ on the sidewalls tends to prevent material removal [47], yielding imperfections [40,45]. Wet etching, on the other hand, can be effective [41,42], although effective masking to prevent undercutting can be problematic, particularly for long etches with small features [48]. Two-dimensional structures etched into lithium niobate have been reported $[42,46]$, however, none have sufficient precision or aspect ratio to enable phononic crystal behavior. Conversely, larger micron-scale phononic crystals have been reported in $\mathrm{LiNbO}_{3}$ [49], but these structures can only achieve phononic band-gap behavior at near- $\mathrm{GHz}$ frequencies, far below the multi-GHz hypersonic frequencies required for strong nanophotonic-phononic interactions.

Recently, we have reported that visible $(\lambda=532 \mathrm{~nm})$ laser irradiation of nanopatterned $\mathrm{Cr}$ can lead to the formation of nanodomains on the surface of a Z-cut $\mathrm{LiNbO}_{3}$ substrate [50]. The regions patterned with $\mathrm{Cr}$ are domain inverted while the uncoated regions remain unaffected by the irradiation. Due to the differential etch rate between inverted and noninverted domains in HF solution, the noninverted domains etch faster than their inverted counterparts. Thus, while the inverted domains at the surface are relatively shallow $(30 \mathrm{~nm})$, they can protect the noninverted material beneath them from etching, thus enabling the formation of high aspect ratio structures, even with un-etched features as small as $100 \mathrm{~nm}$ and etch depths on the order of $200 \mathrm{~nm}$ [50].

In this paper, we show pillar-based hypersonic phononic crystals with nanoscale dimensions exhibiting band gaps at multi-GHz frequencies in lithium niobate. The fabricated phononic crystal consists of a periodic array of nanopillars arranged according to the triangular lattice structure of the crystal and whose phonon band structure is characterized by direct observation using Brillouin light scattering (BLS) [7]. The measured phonon band structure reveals several surface phononic bandgap features, which can be attributed to both local resonances of the pillars and Bragg resonance of the surface phonons across the array. Finite element analysis is used to simulate the expected phonon band structure and transmission spectra of periodic structure, which compare well to the measured results. In particular, the analysis reveals that the measured surface phononic band gaps are complete band gaps that extend throughout the first Brillouin zone and that they are nonradiative and appear below the sound line [13].

\section{MATERIALS AND METHODS}

The fabrication approach is illustrated in Fig. 1. First, we define a 30-nm thin layer of $\mathrm{Cr}$ disk nanopatterns arranged in a triangular lattice covering a $100 \mu \mathrm{m} \times 100 \mu \mathrm{m}$ area using electron beam lithography (EBL) followed by a lift-off process on the $-\mathrm{Z}$ face of a two-side polished $\mathrm{LiNbO}_{3}$ crystal (provided by Gooch \& Housego). The lattice period of the pattern is $a=300 \mathrm{~nm}$ with a duty cycle of 0.4 . The rear face of the crystal is lapped to avoid ambiguity resulting from $2 \alpha \mathrm{A}$ BLS geometry [51]. We then irradiate the $\mathrm{Cr}$ nanopattern by scanning a focused laser beam at wavelength $\lambda=532 \mathrm{~nm}$ across the surface [Fig. 1(a)]. The full process is described in Ref. [50]. Briefly, as the laser is partially absorbed by the $\mathrm{Cr}$, the surface area of $\mathrm{LiNbO}_{3}$ coated with $\mathrm{Cr}$ heats,
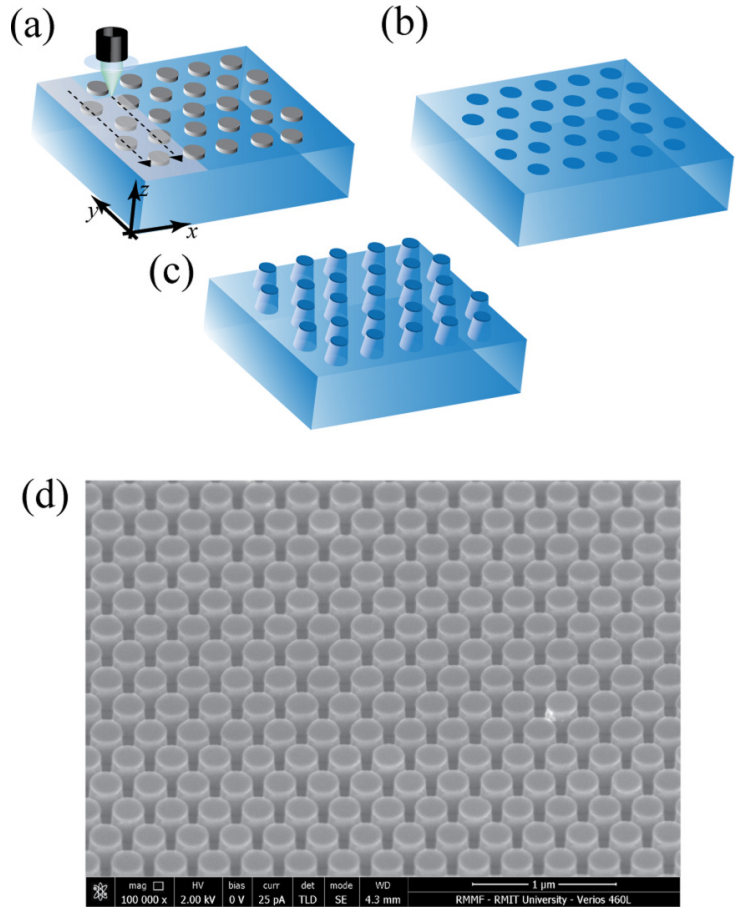

FIG. 1. (a) Schematic illustration of the nanodomain engineering process using laser irradiation in which the domain is written by a focused laser beam at wavelength $\lambda=532 \mathrm{~nm}$ that moves along a direction perpendicular to the $x$ axis. Prior to the laser writing, a $30-\mathrm{nm}$ thin layer of $\mathrm{Cr}$ disk nanopatterns arranged in a triangular lattice is deposited on the $-\mathrm{Z}$ face of the $\mathrm{LiNbO}_{3}$ crystal using EBL. (b) After the process, the $\mathrm{Cr}$ is removed from the sample and the surface of the $\mathrm{LiNbO}_{3}$ is domain inverted to a depth of about $30 \mathrm{~nm}$ [50]. (c) The sample is then etched with $48 \%$ HF solution for $28 \mathrm{~min}$, which leads to the formation of the nanopillars. (d) SEM $45^{\circ}$ angle view image of the fabricated nanopillar phononic crystals in $\mathrm{LiNbO}_{3}$.

which then triggers the domain inversion which rotates the polarization from $-\mathrm{Z}$ to $+\mathrm{Z}$. In contrast, the uncoated area is unaffected by the irradiation since $\mathrm{LiNbO}_{3}$ is transparent at this wavelength. As such, the surface of the $\mathrm{LiNbO}_{3}$ is domain inverted to a depth of about $30 \mathrm{~nm}$ after the removal of the $\mathrm{Cr}$ [50]. The achieved domain pattern is a faithful reproduction of the chrome pattern realized with EBL. The sample is then etched with $48 \% \mathrm{HF}$ solution for $28 \mathrm{~min}$. As the $-\mathrm{Z}$ face is etched much faster than the $+\mathrm{Z}$ face in $\mathrm{HF}$ [41-43], the uninverted region is removed while the inverted domain remains unetched and serves as a mask during the wet etching process. Eventually, the pillar structure is formed [Fig. 1(c)]. The quality of the nanopillars is characterized by scanning electron microscopy (SEM). The $45^{\circ}$-angle view of the achieved periodic nanopillars in Fig. 1(d) shows excellent and uniformly ordered nanopillars with smooth sidewalls as expected. From atomic force microscopy (AFM), the height of the pillar is measured to be $h=225 \mathrm{~nm}$ with diameters $d=240 \mathrm{~nm}$ and $t=170 \mathrm{~nm}$ at the bottom and at the top of the pillar, respectively, which render its shape slight conical (almost cylindrical) with an angle of about $6^{\circ}$ from the normal axis. The relative orientations of the phononic lattice and the $\mathrm{LiNbO}_{3}$ are shown in Fig. 1(a). 
BLS measurements are performed at a room temperature using a six-pass tandem type Fabry-Perot spectrometer (JRS Scientific Instruments), which allows study of thermally activated bulk and surface phonons in the hypersonic range in materials with submicron characteristic length scales [7]. The wavelength of the incident light is $\lambda_{0}=514.5 \mathrm{~nm}\left(\mathrm{Ar}^{+}\right.$laser), and $p$ - $p$ and $p$-s backscattering geometries are used in the measurements. Here, $p$ and $s$ correspond to the polarization of the light to be parallel and normal to the sagittal plane, respectively. The light is focused by an Olympus $10 \times$ long working distance microscope objective with a numerical aperture of 0.45 . The diameter of the laser spot is about a few microns and the power is kept below $3 \mathrm{~mW}$. Given the $\mathrm{LiNbO}_{3}$ substrate is transparent at this wavelength, the sample is coated with a 15-nm thin layer of platinum $(\mathrm{Pt})$ to enhance the surface ripple mechanism in BLS measurements, as illustrated in Fig. 1(c). In the case of surface BLS, momentum conservation is satisfied based on the in-plane components with a magnitude of the scattering vector given by [7]

$$
q=\frac{4 \pi \sin \theta}{\lambda_{0}}
$$

where $\theta$ is the incident (scattered) light angle [Fig. 2(b)]. The phonon dispersion is thus measured by varying the angle $\theta$, allowing one to probe the phonon wave vectors up to the third Brillouin zone (BZ). For periodic structures, the scattering wave vector is defined by momentum conservation, $\mathbf{q}=\mathbf{k}+\mathbf{G} . \mathbf{k}$ is a wave vector in the first BZ as shown by the triangle $K \Gamma M$ in Fig. 1(c), which corresponds to the first irreducible $\mathrm{BZ}$ of the Z-cut $\mathrm{LiNbO}_{3}$ crystal [37], in the k-space representation of the crystal, $\mathbf{G}=l \mathbf{b}_{1}+m \mathbf{b}_{2}$ is the reciprocal lattice vector, in which $\mathbf{b}_{1}$ and $\mathbf{b}_{2}$ are reciprocal

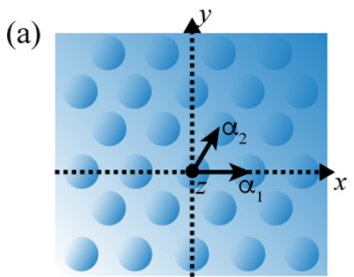

(b)
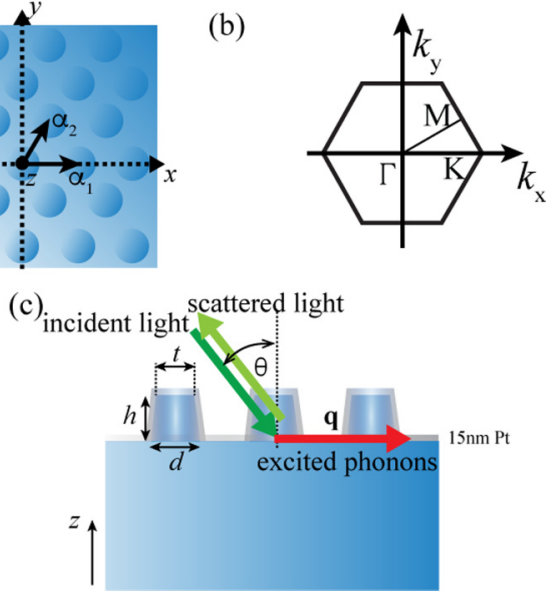

FIG. 2. Schematic of the fabricated hypersonic phononic crystal formed from nanopillars arranged in a triangular lattice. (a) The top view illustrates $\boldsymbol{\alpha}_{1}=(a, 0)$ and $\boldsymbol{\alpha}_{2}=(a / 2, \sqrt{3} a / 2)$ as the principal lattice vectors, with lattice constant $a=\left|\alpha_{1}\right|=\left|\alpha_{2}\right|$. (b) $2 \mathrm{D}$ reciprocal space for the triangular lattice. (c) The lateral view shows the characteristic sizes of the pillars and the geometry of the Brillouin light scattering in which $h, d$, and $t$ denote the height, bottom diameter, and top diameter of the pillars, respectively. Prior to the BLS measurement, the surface is coated with a 15 -nm thin layer of Pt to optimize the BLS signal; q denotes the scattering wave vectors and $\theta$ the scattering angle. unit vectors, and $l$ and $m$ are integers. The unit vectors $\mathbf{b}_{1,2}$ are calculated from the real-space basic vectors, $\boldsymbol{\alpha}_{1}=(a, 0)$ and $\boldsymbol{\alpha}_{2}=(a / 2, \sqrt{3} a / 2)$ for the triangular lattice illustrated in Fig. 1(a). BLS measurements are carried out for scattering directions along $x$ and $y$ directions for several angles $\theta$ from $18^{\circ}$ to $60^{\circ}$ and $40^{\circ}$ to $60^{\circ}$. The largest scattering is limited to $60^{\circ}$ as this gives the scattering wave vector $\mathbf{q}$ that follows the edge of the triangle $K \Gamma M \Gamma$ in Fig. 1(c). Consequently, a complete picture of the phonon band structure of the phononic crystal can be plotted, from which the existence of complete phononic band gaps can be determined.

\section{RESULTS AND DISCUSSION}

Figures 3(a) and 3(b) show the BLS spectra at $q=$ $0.00915 \mathrm{~nm}^{-1}$ in the $x$ direction $(\Gamma K)$ measured in the $p$ - $p$ and $p$ - $s$ polarizations. Stokes and anti-Stokes components located at $\mathrm{A}, \mathrm{B}, \mathrm{C}$, and $\mathrm{D}$ can be identified in the figure that directly indicates the measured phonon modes of the phononic crystal. Modes located at peaks B, C, and D from the $p-p$ geometry in Fig. 3(a) correspond to phonon modes whose polarization is parallel to the sagittal plane, which is the $z-x$ plane for the surface phonons propagating along the $x$ axis, whereas the mode at peak A measured in the $p-s$ geometry in Fig. 3(b) possess polarization normal to the sagittal plane.

To further verify the characteristics of the observed phonon modes, finite element analysis (FEA) is performed. In the simulation, the thin layer of Pt is included; the bulk material properties of $\mathrm{LiNbO}_{3}$ and $\mathrm{Pt}$ are taken from the material library provided in COMSOL Multiphysics where mass density of the lithium niobate, $\rho=4700 \mathrm{~kg} \mathrm{~m}^{-3}$, and $\mathrm{Pt}$, $\rho=21450 \mathrm{~kg} \mathrm{~m}^{-3}$. The calculated displacement profiles of the modes associated with peaks A-D are shown in Figs. 3(c)-3(f). From the displacement profiles, it can be deduced that all the

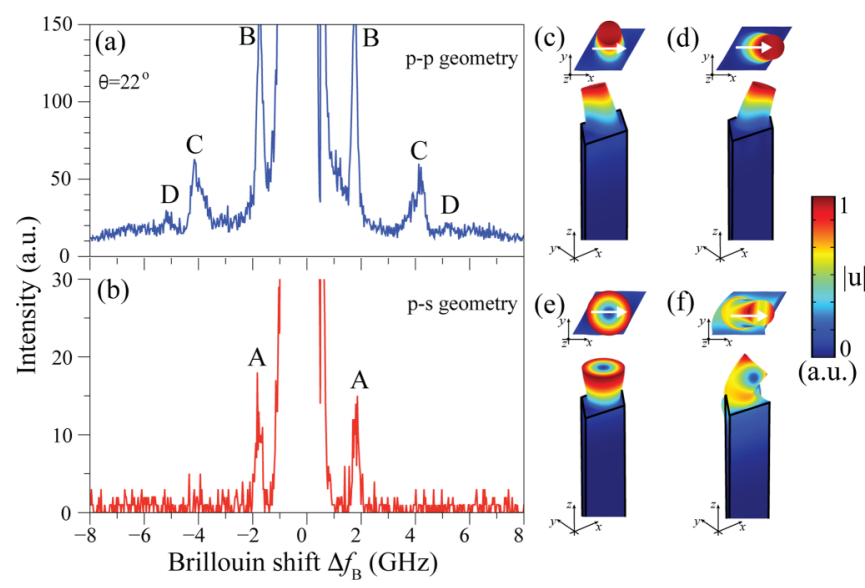

FIG. 3. BLS spectra for a scattering angle $\theta=22^{\circ}$ corresponding to $q=0.00915 \mathrm{~nm}^{-1}$ obtained for $\Gamma K$ or the $x$ direction for (a) the $p$ - $p$ geometry, and, (b) the $p-s$ geometry. The right panel shows the calculated deformation of the surface phonon profiles associated with (c) peak A (oscillating along the cartesian $y$ axis), (d) peak $\mathrm{B}$ (oscillating along the cartesian $x$ axis), (e) peak $\mathrm{C}$ (breathing oscillation), and (f) peak D (hybrid surface wave); the white arrow indicates the propagation direction along the $x$ axis on the sagittal plane or the $z-x$ plane in the measurements. 
observed phonon modes correspond to surface phonons with their displacements mostly confined on the surface substrate. Figures 3(c)-3(f) also show that the mode associated with peak $A$ at $1.86 \mathrm{GHz}$ has polarization perpendicular to the sagittal plane, whereas the other modes for peaks B, C, and $\mathrm{D}$ at $1.75,4.15$, and $5.18 \mathrm{GHz}$ have polarization mostly on the sagittal plane, which confirm the measurement results. In addition, we can see from the calculated displacement profiles that the modes associated with clear and sharp Brillouin signal at peaks A, B, and C in Figs. 3(a) and 3(b) correspond to surface phonon modes associated with local resonance of the pillar; the elastic energy is mostly confined within the pillars. On the contrary, the less intense signal at peak D corresponds to a hybrid surface phonon mode that resembles a Rayleigh surface wave. We interpret that the confinement of the elastic energy within the pillars of the locally resonant phonon modes gives rise to a clear and sharp measured Brillouin signal whereas the signal associated with the Rayleigh surface modes is mostly distributed on the substrate surface.

BLS measurements performed for different angles $\theta$ along the $x$ and $y$ axes are plotted in Figs. 4(a) and 4(b), respectively, as the frequencies of the measured phonon modes are plotted against the reduced wave vector, indicated by the solid circles. When the incident angle $\theta \geqslant 35^{\circ}$ for the measurements in the $x$ direction, the scattering vector, with magnitude $q=$ $0.01401 \mathrm{~nm}^{-1}$, is located outside the boundary of the first BZ at point $K, k=4 \pi / 3 a=0.01396 \mathrm{~nm}^{-1}$. Through the reciprocal lattice vector $\mathbf{G}$ all the scattering vectors are translated to the first BZ, which corresponds to wave vectors in the $K M$ direction, as illustrated in the inset of Fig. 4(a). Given the symmetry of the slowness curve of the $\mathrm{Z}$-cut $\mathrm{LiNbO}_{3}$ crystal [37], the phonon modes measured along the $y$ direction are identical to the modes in the $M \Gamma$ direction in the first irreducible BZ, as plotted in Fig. 4(b).

To better understand the characteristics of the observed phononic dispersion, we carry out numerical simulations using FEA to calculate the phonon band structures, and the transmission spectra along the highest point in the BZ, namely, $\Gamma K$ and $\Gamma M$, corresponding to the $x$ and $y$ axes, respectively. Band structure and transmission computations are performed following the procedures explained in Refs. [3,37]. Briefly, two finite sizes of phononic crystal structures are considered, namely, eight-cell and 10-cell for $\Gamma K$ and $\Gamma M$, respectively. A sagittally polarized surface phonon source excited by an interdigital electrode is considered for the transmission calculation. The calculated phonon band structures are plotted in Figs. 4(a) and 4(b) superposed onto the measured structures, and the transmission spectra for $\Gamma K$ and $\Gamma M$ directions are plotted in Figs. 4(c) and 4(d), respectively. The calculated phonon dispersion (blue dots) is shown below the sound line (red line) ensuring the nonradiative character of the branches plotted outside the gray area. The sound line is determined from the transverse bulk acoustic velocities calculated by taking into account the $15-\mathrm{nm}$ thin layer of $\mathrm{Pt}$, which are $3500 \mathrm{~m} \mathrm{~s}^{-1}$ and $3283 \mathrm{~m} \mathrm{~s}^{-1}$ for $\Gamma K$, and $\Gamma K$, respectively.

We can perceive in Figs. 4(a) and 4(b) the flatness of the branches apearing below $4.5 \mathrm{GHz}$, with good agreement between experimental and simulated dispersion relation. The observed nondispersive branches at $1.8 \mathrm{GHz}$ and $4 \mathrm{GHz}$ are typically due to locally resonant modes [3,4,7], which
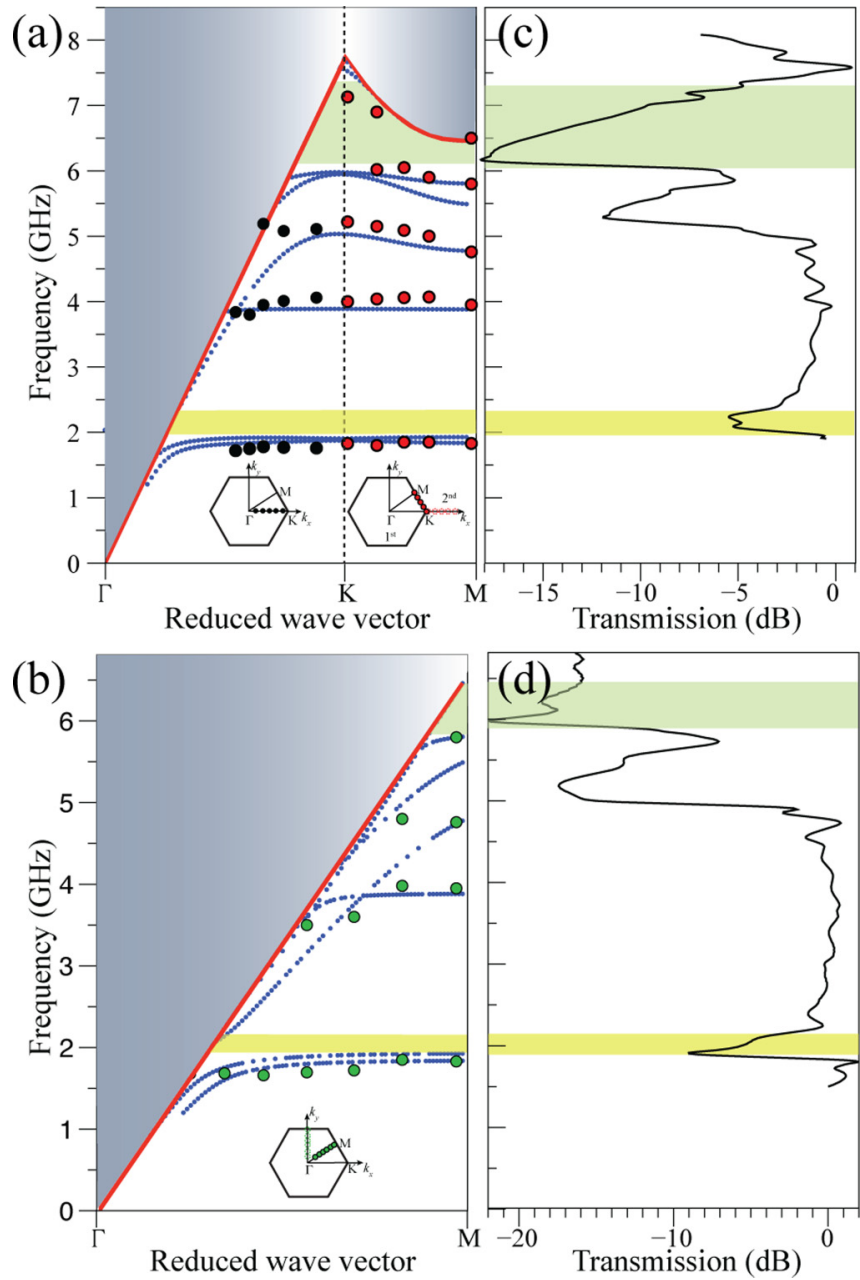

FIG. 4. Experimentally measured phonon band structures for (a) the $\Gamma K$ and $K M$ directions, represented by the solid black and red circles, respectively, and (b) the $\Gamma M$ direction shown by the solid green circles, superposed with the calculated phononic band structures denoted by blue dots. (c) and (d) Theoretical transmission spectra for the surface phonons propagating in the $x$ and $y$ directions, respectively. The yellow and green regions denote the locally resonant and Bragg surface phononic band gaps, respectively. The red lines represent the sound line of the $\mathrm{LiNbO}_{3}$ substrate taking into account the 15-nm thin layer of $\mathrm{Pt}$ whereas the gray areas represent the substrate or leaky modes. (Insets) Positions of the wave vector $(q)$ from the BLS measurement in the $2 \mathrm{D}$ reciprocal space following the edge of the triangle $K \Gamma M$ within the irreducible $\mathrm{BZ}$.

correspond to deformations of the pillars as shown in Fig. 3. The branches at higher frequency, on the other hand, appear to be more dispersive due possibly to the interaction of local resonant modes and surface propagating modes. Furthermore, by comparing the experimental and numerical results in Figs. 4(a)-4(d), two band gaps can be observed. The first band gap represented by the yellow region appears at a frequency of around $2 \mathrm{GHz}$ located at the far side of the BZ boundary and above the flat bands. This band gap is delimited by the attenuation frequency range in the transmission spectra, which results from the hybridization between the local resonances at the pillars and the Rayleigh propagating modes of the surface. The strong dispersion seen close to the sound line actually 




FIG. 5. Experimentally and calculated phonon band structure of the fabricated nanopillar-based phononic crystal along the edge of the triangle $K \Gamma M$ within the irreducible BZ, which reveals complete surface phononic band gaps that are attributed to locally resonant and Bragg resonant surface phononic band gaps at a frequency of around $2 \mathrm{GHz}$ and $7 \mathrm{GHz}$, respectively. The insets show mode profiles of the surface phonons forming the branch located at around $5 \mathrm{GHz}$ with polarization perpendicular to the sagittal plane with the white arrows indicating the propagation directions.

results from the anticrossing of the branches related to these two different modes, indicating the strong mixing and gradual conversion from purely local modes to purely propagative Rayleigh modes. The second band gap, on the other hand, appears at a frequency of around $7 \mathrm{GHz}$, as indicated by the green region, close to the BZ boundary, and thus can be attributed to Bragg scattering. The formation of the Bragg band gap is as expected, as the presented phononic crystal is an ordered structure formed from an array of pillars of hexagonal lattice [4]. In the case of a disordered structure, however, Bragg scattering gap should be absent because the phase matching condition for Bragg scattering could not be satisfied [52].

The results reported in Figs. 4(a) and 4(b) therefore provide clear evidence of the existence of simultaneous locally resonant and Bragg surface phononic band gaps in the fabricated nanopillar phononic crystal within the hypersonic multi- $\mathrm{GHz}$ frequency region. Despite the minimum transmission at around $5 \mathrm{GHz}$ observed in the transmission spectra in Figs. 4(c) and 4(d), this region, however, is not attributed to a band gap because of the presence of a branch within the region [see Figs. 4(a) and 4(b)]. Here, the attenuation is caused as this branch is deaf to the sagittal source [3]; the polarization of the surface modes that manifest the branch is perpendicular to the sagittal plane as clearly shown by the insets in Fig. 5. Unlike the deaf mode in Ref. [6], theoretically, these modes can be excited with a shear horizontally polarized excitation line source [3]. The absence of features related to the branch about $2.1 \mathrm{GHz}$ and between 5.5 GHZ and $6 \mathrm{GHz}$ in BLS spectra for both $p$ - $p$ and $p$-s, may be attributed to weak or null out-of-plane displacements [7].

By combining the measured and calculated phonon band structures in Figs. 4(a) and 4(b) into a single plot in Fig. 5, a complete representation of the phononic band structure of the crystal within the irreducible BZ can be achieved. The horizontal dashed line in Fig. 5 is defined by the lowest frequency attained by a bulk wave with its vector exactly on the BZ boundary. We can clearly see from Fig. 5 that both locally resonant and Bragg surface phononic band gaps are complete band gaps and hence omnidirectional as they extend throughout the BZ: The complete locally resonant surface phononic band gap arises between $1.9 \mathrm{GHz}$ and $2.05 \mathrm{GHz}$ whereas the second complete surface phononic band gap due to the Bragg resonance appears in the range between 5.97 and $6.5 \mathrm{GHz}$. In addition, the existence of a directional Bragg band gap above the dashed line can be seen to extend from 6.5 to $7.0 \mathrm{GHz}$, which is deduced based on its location with respect to the sound line within the first Brillouin zone [13]. As all the observed band gaps are found below the sound line, they are hence nonradiative [13]. The presence of Pt thin film and its impact on the phononic behavior should also be noted: Despite its 15-nm thickness, its density, which is five times higher than that of $\mathrm{LiNbO}_{3}$ leads to the introduction of a mass-loading effect, which we estimate to shift the frequency by approximately $0.5 \mathrm{GHz}$ for all bands.

\section{CONCLUSIONS}

We have experimentally demonstrated the first pillar-based hypersonic phononic crystal with nanoscale dimensions in single crystal Z-cut $\mathrm{LiNbO}_{3}$ that exhibits band gaps at multi$\mathrm{GHz}$ frequencies. The nanopillars are fabricated by a recently introduced technique involving nanodomain engineering via laser irradiation of patterned $\mathrm{Cr}$ followed by wet etching. The experimentally observed surface phononic band gap are verified by numerical modeling to be complete, nonradiative band gaps that extend throughout the first Brillouin zone. In addition to potentially opening new frontiers in research in acousto-nanofluidic manipulation and eventually thermoelectric structures for energy harvesting, this work represents a significant step towards monolithic integration of photonics and phononics on the industry standard $\mathrm{LiNbO}_{3}$ platform. Our future research will thus focus on interfacing these phononic structures with nanophotonic and plasmonic arrays, exploiting the overlap in critical dimensions of these hypersonic phononic crystals and their photonic counterparts to achieve strong photon-phonon interactions.

\section{ACKNOWLEDGMENTS}

Nanodomain engineering in this work was carried out at the Melbourne Centre for Nanofabrication (MCN) and the MicroNano Research Facility (MNRF) at RMIT Univeristy. The technique for nanodomain engineering was a scientific output of the ARC Centre of Excellence program (CE110001018). B.G., F.A., and C.M.S.T. acknowledge the partial support of the Spanish national projects PHENTOM (FIS2015-70862-P) and the Severo Ochoa program (Grant No. SEV-2013-0295) and the EU projects MERGING (Grant No. 309150).
[1] Z. Liu, X. Zhang, Y. Mao, Y. Y. Zhu, Z. Yang, C. T. Chan, and P. Sheng, Science 289, 1734 (2000).
[2] T. Still, W. Cheng, M. Retsch, R. Sainidou, J. Wang, U. Jonas, N. Stefanou, and G. Fytas, Phys. Rev. Lett. 100, 194301 (2008). 
[3] A. Khelif, Y. Achaoui, S. Benchabane, V. Laude, and B. Aoubiza, Phys. Rev. B 81, 214303 (2010).

[4] Y. Achaoui, A. Khelif, S. Benchabane, L. Robert, and V. Laude, Phys. Rev. B 83, 104201 (2011).

[5] F. Lemoult, N. Kaina, M. Fink, and G. Lerosey, Nat. Phys. 9, 55 (2013).

[6] E. Alonso-Redondo, M. Schmitt, Z. Urbach, C. M. Hui, R. Sainidou, P. Rembert, K. Matyjaszewski, M. R. Bockstaller, and G. Fytas, Nat. Commun. 6, 8309 (2015).

[7] B. Graczykowski, M. Sledzinska, F. Alzina, J. Gomis-Bresco, J. S. Reparaz, M. R. Wagner, and C. M. Sotomayor Torres, Phys. Rev. B 91, 075414 (2015).

[8] W. Cheng, J. Wang, U. Jonas, G. Fytas, and N. Stefanou, Nat. Mater. 5, 830 (2006),

[9] T. Gorishnyy, C. K. Ullal, M. Maldovan, G. Fytas, and E. L. Thomas, Phys. Rev. Lett. 94, 115501 (2005).

[10] T.-T. Wu, Z. G. Huang, and S. Lin, Phys. Rev. B 69, 094301 (2004).

[11] S. Benchabane, A. Khelif, J.-Y. Rauch, L. Robert, and V. Laude, Phys. Rev. E 73, 065601 (2006).

[12] J. O. Vasseur, P. A. Deymier, B. Djafari-Rouhani, Y. Pennec, and A. C. Hladky-Hennion, Phys. Rev. B 77, 085415 (2008).

[13] D. Yudistira, Y. Pennec, B. Djafari-Rouhani, S. Dupont, and V. Laude, Appl. Phys. Lett. 100, 061912 (2012).

[14] L. Feng, X.-P. Liu, Y.-B. Chen, Z.-P. Huang, Y.-W. Mao, Y.-F. Chen, J. Zi, and Y.-Y. Zhu, Phys. Rev. B 72, 033108 (2005).

[15] B. Bonello, L. Belliard, J. Pierre, J. O. Vasseur, B. Perrin, and O. Boyko, Phys. Rev. B 82, 104109 (2010).

[16] A. Sukhovich, L. Jing, and J. H. Page, Phys. Rev. B 77, 014301 (2008).

[17] P. H. Otsuka, K. Nanri, O. Matsuda, M. Tomoda, D. M. Profunser, I. A. Veres, S. Danworaphong, A. Khelif, S. Benchabane, V. Laude et al., Sci. Rep. 3, 3351 (2013).

[18] M. Maldovan and E. L. Thomas, Appl. Phys. Lett. 88, 251907 (2006).

[19] M.-H. Lu, L. Feng, and Y.-F. Chen, Mater. Today 12, 34 (2009).

[20] R. H. Olsson III and I. El-Kady, Meas. Sci. Technol. 20, 012002 (2009).

[21] A. H. Safavi-Naeini, J. T. Hill, S. Meenehan, J. Chan, S. Gröblacher, and O. Painter, Phys. Rev. Lett. 112, 153603 (2014).

[22] H. Li, S. A. Tadesse, Q. Liu, and M. Li, Optica 2, 826 (2015).

[23] K. C. Balram, M. I. Davanço, J. D. Song, and K. Srinivasan, Nat. Photon. 10, 346 (2016).

[24] J. Gomis-Bresco, D. Navarro-Urrios, M. Oudich, S. El-Jallal, A. Griol, D. Puerto, E. Chavez, Y. Pennec, B. Djafari-Rouhani, F. Alzina et al., Nat. Commun. 5, 4452 (2014).

[25] C. Ruppert, J. Neumann, J. B. Kinzel, H. J. Krenner, A. Wixforth, and M. Betz, Phys. Rev. B 82, 081416 (2010).

[26] J. Schiefele, J. Pedrós, F. Sols, F. Calle, and F. Guinea, Phys. Rev. Lett. 111, 237405 (2013).

[27] T. A. Kelf, W. Hoshii, P. H. Otsuka, H. Sakuma, I. A. Veres, R. M. Cole, S. Mahajan, J. J Baumberg, M. Tomoda, O. Matsuda et al., New J. Phys. 15, 023013 (2013).
[28] M. Maldovan, Nature (London) 503, 209 (2013).

[29] P. E. Hopkins, C. M. Reinke, M. F. Su, I. Roy H. Olsson, E. A. Shaner, Z. C. Leseman, J. R. Serrano, L. M. Phinney, and I. El-Kady, Nano Lett. 11, 107 (2011).

[30] J.-K. Yu, S. Mitrovic, D. Tham, J. Varghese, and J. R. Heath, Nat. Nano. 5, 718 (2010).

[31] D. Bolmatov, M. Zhernenkov, D. Zav'yalov, S. Stoupin, A. Cunsolo, and Y. Q. Cai, Sci. Rep. 6, 19469 (2016).

[32] R. J. Shilton, M. Travagliati, F. Beltram, and M. Cecchini, Adv. Mater. 26, 4941 (2014).

[33] M. K. Tan, L. Y. Yeo, and J. R. Friend, EPL 87, 47003 (2009).

[34] B. Graczykowski, S. Mielcarek, A. Trzaskowska, J. Sarkar, P. Hakonen, and B. Mroz, Phys. Rev. B 86, 085426 (2012).

[35] M. Kösters, B. Sturman, P. Werheit, D. Haertle, and K. Buse, Nat. Photonics 3, 510 (2009).

[36] C. Campbell, Surface Acoustic Wave Devices and Their Signal Processing Applications (Elsevier, Amsterdam, 2012).

[37] D. Yudistira, A. Boes, B. Djafari-Rouhani, Y. Pennec, L. Y. Yeo, A. Mitchell, and J. R. Friend, Phys. Rev. Lett. 113, 215503 (2014).

[38] D. Yudistira, A. Boes, B. Dumas, A. R. Rezk, M. Yousefi, B. Djafari-Rouhani, L. Y. Yeo, and A. Mitchell, Ann. Phys. (Berlin) 528, 365 (2016).

[39] J. Reboud, Y. Bourquin, R. Wilson, G. S. Pall, M. Jiwaji, A. R. Pitt, A. Graham, A. P. Waters, and J. M. Cooper, Proc. Natl. Acad. Sci. USA 109, 15162 (2012).

[40] A. Lecestre, S. Benchabane, L. Robert, R. Salut, G. Ulliac, and P. Blind, Microelectron. Eng. 105, 95 (2013).

[41] I. E. Barry, G. W. Ross, P. G. Smith, R. W. Eason, and G. Cook, Mater. Lett. 37, 246 (1998).

[42] S. Mailis, C. Sones, and R. Eason, Ferroelectric Crystals for Photonic Applications (Springer, Berlin, 2014), pp. 3-19.

[43] C. L. Sones, S. Mailis, W. S. Brocklesby, R. W. Eason, and J. R. Owen, J. Mater. Chem. 12, 295 (2002).

[44] F. Lacour, N. Courjal, M.-P. Bernal, A. Sabac, C. Bainier, and M. Spajer, Opt. Mater. 27, 1421 (2005).

[45] S. Benchabane, L. Robert, J.-Y. Rauch, A. Khelif, and V. Laude, J. Appl. Phys. 105, 094109 (2009).

[46] G. Ulliac, B. Guichardaz, J.-Y. Rauch, S. Queste, S. Benchabane, and N. Courjal, Microelectron. Eng. 88, 2417 (2011).

[47] H. Nagata, N. Mitsugi, K. Shima, M. Tamai, and E. Haga, J. Cryst. Growth 187, 573 (1998).

[48] H. Hui, R. Ricken, and W. Sohler, ECIO Eindhoven (IEEE, New York, 2008), pp. 75-78.

[49] S. Benchabane, O. Gaiffe, G. Ulliac, R. Salut, Y. Achaoui, and V. Laude, Appl. Phys. Lett. 98, 171908 (2011).

[50] A. Boes, V. Sivan, G. Ren, D. Yudistira, S. Mailis, E. Soergel, and A. Mitchell, Appl. Phys. Lett. 107, 022901 (2015).

[51] J. K. Krüger, J. Embs, J. Brierley, and R. Jiménez, J. Phys. D 31, 1913 (1998).

[52] Y. Achaoui, V. Laude, S. Benchabane, and A. Khelif, J. Appl. Phys. 114, 104503 (2013). 